\title{
ON POINCARÉ TYPE INTEGRAL INEQUALITIES
}

\author{
WING-SUM CHEUNG
}

(Communicated by Andrew Bruckner)

\begin{abstract}
In this paper some new integral inequalities of the Poincare type, on a region of rectangular dimensions, involving many functions in many variables are obtained. These in turn can be used to serve as generators of other integral inequalities.
\end{abstract}

\section{INTRODUCTION}

The multidimensional integral inequality $[4,7]$

$$
\lambda_{0} \int_{\Omega} u^{2} d x \leq \int_{\Omega}|\nabla u|^{2} d x
$$

where $\Omega$ is a bounded region in $\mathbb{R}^{2}$ or $\mathbb{R}^{3}, u \in C^{1}(\Omega), u=0$ on $\partial \Omega$, and $\lambda_{0}$ is the smallest eigenvalue of the problem

$$
\begin{cases}\Delta u+\lambda u=0 & \text { in } \Omega, \\ u=0 & \text { on } \partial \Omega,\end{cases}
$$

is known as Poincaré's inequality. Since (1) plays a fundamental role in the theory as well as applications of partial differential equations, considerable attention and effort have been put on its generalizations to various aspects. For details one is referred to Beckenbach and Bellman [1], Hardy, Littlewood, and Pólya [3], Mitrinović [7], Nirenberg [8], and, more recently, Horgan [4-6], Pachpatte $[9,10]$, and Cheung [2]. It is the purpose of this paper to obtain some new generalizations of inequality (1) in the context involving many functions in many variables by methods which are rather elementary compared to the usual technique of considering certain eigenvalue problems.

\section{NOTATION AND PRELIMINARIES}

Throughout this paper, let $m \geq 2$ and $n \geq 1$ be any two fixed integers. Let $\alpha, \beta$ be indices running from 1 to $m$, and $i, j$ from 1 to $n$. Let $\Omega=\prod_{i=1}^{n}\left[a_{i}, b_{i}\right] \subset \mathbb{R}^{n}$ be a fixed rectangular region. A general point in $\Omega$ will be denoted by $x=\left(x_{1}, \ldots, x_{n}\right)$, and the volume form will be denoted by $d x=d x_{1} \cdots d x_{n}$. Let $C_{0}^{1}(\Omega)$ be the collection of all real-valued continuously

Received by the editors March 11, 1992.

1991 Mathematics Subject Classification. Primary 26D10, 26D15, 39B72.

Key words and phrases. Poincaré inequality. 
differentiable functions on $\Omega$ which vanish on the boundary $\partial \Omega$ of $\Omega$. As usual, partial derivatives of $f \in C_{0}^{1}(\Omega)$ will be denoted by $f_{i}$.

Since all summations and products appearing in this paper will be either over $\alpha, \beta$ or $i, j$, and hence should be from 1 to $m$ or from 1 to $n$, respectively, for the sake of simplicity we shall drop the terminals of the summation sign $\sum$ and the product sign $\Pi$ unless confusion may occur. Furthermore, as usual, integrals of the form $\int_{\Omega} F(x) d x$ will be abbreviated by $\int_{\Omega} F$.

The following basic inequalities will be needed in the sequel.

Inequality $\mathbf{A}$ [7]. For any $p_{\alpha}>0$ with $\sum_{\alpha} 1 / p_{\alpha}=1$, and for any $c_{\alpha} \geq 0$,

$$
\prod_{\alpha} c_{\alpha} \leq \sum_{\alpha} \frac{1}{p_{\alpha}} c_{\alpha}^{p_{\alpha}} \text {. }
$$

Inequality B [3]. For any $q_{\alpha}>0$ and $c_{\alpha} \geq 0$,

$$
\prod_{\alpha} c_{\alpha}^{q_{\alpha}} \leq \frac{1}{q} \sum_{\alpha} q_{\alpha} c_{\alpha}^{q}
$$

where $q:=\sum_{\beta} q_{\beta}$.

Inequality $\mathbf{C}$ [7]. For any $r_{i} \geq 0$ and $s \geq 0$,

$$
\left(\sum_{i} r_{i}\right)^{s} \leq c(s, n) \sum_{i} r_{i}^{s}
$$

where

$$
c(s, n)= \begin{cases}n^{s-1} & \text { if } s>1, \\ 1 & \text { if } 0 \leq s \leq 1 .\end{cases}
$$

Inequality $\mathbf{D}$ (Generalization of Hölder's inequality). For any $p_{\alpha}>0$ with $\sum_{\alpha} 1 / p_{\alpha}=1$ and any $g^{\alpha} \in \mathscr{L}^{p_{\alpha}}(\Omega)$, we have $\prod_{\alpha} g^{\alpha} \in \mathscr{L}^{1}(\Omega)$ and

$$
\int_{\Omega} \prod_{\alpha}\left|g^{\alpha}\right| \leq \prod_{\alpha}\left(\int_{\Omega}\left|g^{\alpha}\right|^{p_{\alpha}}\right)^{1 / p_{\alpha}} .
$$

Inequality $\mathrm{D}$ is a simple generalization of the classical Hölder's inequality to the case involving more than two functions, which can be proved easily by induction.

\section{MAIN ReSUlts}

Let $M=\max \left\{b_{i}-a_{i}: i=1, \ldots, n\right\}$.

Theorem 1. For any $f^{\alpha} \in C_{0}^{1}(\Omega)$ and any real numbers $p_{\alpha} \geq 2$ satisfying $\sum_{\alpha} 1 / p_{\alpha}=1$,

$$
\int_{\Omega} \prod_{\alpha}\left|f^{\alpha}\right| \leq \frac{1}{n} \sum_{\alpha} \frac{1}{p_{\alpha}}\left(\frac{M}{2}\right)^{p_{\alpha}} \int_{\Omega}\left|\nabla f^{\alpha}\right|^{p_{\alpha}} .
$$

Theorem 2. For any $f^{\alpha} \in C_{0}^{1}(\Omega)$ and any real numbers $q_{\alpha}>0$ with $q:=$ $\sum_{\alpha} q_{\alpha} \geq 2$,

$$
\int_{\Omega} \prod_{\alpha}\left|f^{\alpha}\right|^{q_{\alpha}} \leq \frac{1}{n}\left(\frac{M}{2}\right)^{q} \sum_{\alpha} \frac{q_{\alpha}}{q} \int_{\Omega}\left|\nabla f^{\alpha}\right|^{q} .
$$


Note that these results generalize the existing Poincare type integral inequalities $[6,7,9,10]$ in the literature. To prove these, we first observe the following

Lemma 1. If $f \in C_{0}^{1}(\Omega)$, then, for any $t \in \Omega$,

$$
|f(t)| \leq \frac{1}{2 n} \sum_{i} \int_{a_{i}}^{b_{i}}\left|f_{i}\left(t_{1}, \ldots, t_{i-1}, u_{i}, t_{i+1}, \ldots, t_{n}\right)\right| d u_{i} .
$$

Proof. For each $i$ we have

$$
f(t)=\int_{a_{i}}^{t_{i}} f_{i}\left(t_{1}, \ldots, t_{i-1}, u_{i}, t_{i+1}, \ldots, t_{n}\right) d u_{i}
$$

and also

$$
f(t)=-\int_{t_{i}}^{b_{i}} f_{i}\left(t_{1}, \ldots, t_{i-1}, u_{i}, t_{i+1}, \ldots, t_{n}\right) d u_{i} .
$$

Taking their absolute values and summing with respect to $i$, we then have

$$
2 n|f(t)| \leq \sum_{i} \int_{a_{i}}^{b_{i}}\left|f_{i}\left(t_{1}, \ldots, t_{i-1}, u_{i}, t_{i+1}, \ldots, t_{n}\right)\right| d u_{i}
$$

and thus the assertion follows. Q.E.D.

Proof of Theorem 1. By Inequality A, Lemma 1, and Inequality C, we have

$$
\begin{aligned}
\prod_{\alpha}\left|f^{\alpha}(t)\right| & \leq \sum_{\alpha} \frac{1}{p_{\alpha}}\left|f^{\alpha}(t)\right|^{p_{\alpha}} \leq \sum_{\alpha} \frac{1}{p_{\alpha}}\left[\frac{1}{2 n} \sum_{i} \int_{a_{i}}^{b_{i}}\left|f_{i}^{\alpha}\right| d u_{i}\right]^{p_{\alpha}} \\
& \leq \sum_{\alpha} \frac{1}{p_{\alpha}}\left(\frac{1}{2 n}\right)^{p_{\alpha}}\left[c\left(p_{\alpha}, n\right) \sum_{i}\left(\int_{a_{i}}^{b_{i}}\left|f_{i}^{\alpha}\right| d u_{i}\right)^{p_{\alpha}}\right] .
\end{aligned}
$$

Since $p_{\alpha}>1$, we have $c\left(p_{\alpha}, n\right)=n^{p_{\alpha}-1}$ for all $\alpha$ and so, by Hölder's inequality,

$$
\begin{aligned}
\prod_{\alpha}\left|f^{\alpha}(t)\right| & \leq \sum_{\alpha} \frac{1}{n p_{\alpha}}\left(\frac{1}{2}\right)^{p_{\alpha}} \sum_{i}\left(\int_{a_{i}}^{b_{i}}\left|f_{i}^{\alpha}\right| d u_{i}\right)^{p_{\alpha}} \\
& \leq \sum_{\alpha} \frac{1}{n p_{\alpha}}\left(\frac{1}{2}\right)^{p_{\alpha}} \sum_{i}\left[\left(b_{i}-a_{i}\right)^{\left(p_{\alpha}-1\right) / p_{\alpha}}\left(\int_{a_{i}}^{b_{i}}\left|f_{i}^{\alpha}\right|^{p_{\alpha}} d u_{i}\right)^{1 / p_{\alpha}}\right]^{p_{\alpha}} \\
& \leq \sum_{\alpha} \frac{1}{n p_{\alpha}}\left(\frac{1}{2}\right)^{p_{\alpha}} M^{p_{\alpha}-1} \sum_{i} \int_{a_{i}}^{b_{i}}\left|f_{i}^{\alpha}\right|^{p_{\alpha}} d u_{i}
\end{aligned}
$$


Therefore,

$$
\begin{aligned}
\int_{\Omega} \prod_{\alpha}\left|f^{\alpha}(t)\right| d t & \leq \sum_{\alpha} \frac{1}{n p_{\alpha}}\left(\frac{1}{2}\right)^{p_{\alpha}} M^{p_{\alpha}-1} \sum_{i} \int_{\Omega} \int_{a_{i}}^{b_{i}}\left|f_{i}^{\alpha}\right|^{p_{\alpha}} d u_{i} d t \\
& =\sum_{\alpha} \frac{1}{n p_{\alpha}}\left(\frac{1}{2}\right)^{p_{\alpha}} M^{p_{\alpha}-1} \sum_{i} \int_{a_{i}}^{b_{i}} \int_{\Omega}\left|f_{i}^{\alpha}\right|^{p_{\alpha}} d t d u_{i} \\
& =\sum_{\alpha} \frac{1}{n p_{\alpha}}\left(\frac{1}{2}\right)^{p_{\alpha}} M^{p_{\alpha}-1} \sum_{i}\left(\int_{\Omega}\left|f_{i}^{\alpha}\right|^{p_{\alpha}} d t\right)\left(b_{i}-a_{i}\right) \\
& \leq \sum_{\alpha} \frac{1}{n p_{\alpha}}\left(\frac{M}{2}\right)^{p_{\alpha}} \int_{\Omega} \sum_{i}\left|f_{i}^{\alpha}\right|^{p_{\alpha}} d t \\
& =\sum_{\alpha} \frac{1}{n p_{\alpha}}\left(\frac{M}{2}\right)^{p_{\alpha}} \int_{\Omega}\left[\left(\sum_{i}\left|f_{i}^{\alpha}\right|^{p_{\alpha}}\right)^{2 / p_{\alpha}}\right]^{p_{\alpha} / 2} d t \\
& \leq \sum_{\alpha} \frac{1}{n p_{\alpha}}\left(\frac{M}{2}\right)^{p_{\alpha}} \int_{\Omega}\left[c\left(\frac{2}{p_{\alpha}}, n\right) \sum_{i}\left(\left|f_{i}^{\alpha}\right|^{p_{\alpha}}\right)^{2 / p_{\alpha}}\right]^{p_{\alpha} / 2} d t
\end{aligned}
$$

by Inequality C. Since $p_{\alpha} \geq 2, c\left(\frac{2}{p_{\alpha}}, n\right)=1$ for all $\alpha$ and so

$$
\begin{aligned}
\int_{\Omega} \prod_{\alpha}\left|f^{\alpha}(t)\right| d t & \leq \sum_{\alpha} \frac{1}{n p_{\alpha}}\left(\frac{M}{2}\right)^{p_{\alpha}} \int_{\Omega}\left(\sum_{i}\left|f_{i}^{\alpha}\right|^{2}\right)^{p_{\alpha} / 2} d t \\
& =\frac{1}{n} \sum_{\alpha} \frac{1}{p_{\alpha}}\left(\frac{M}{2}\right)^{p_{\alpha}} \int_{\Omega}|\nabla f|^{p_{\alpha}} d t \text {. Q.E.D. }
\end{aligned}
$$

Proof of Theorem 2. By Inequality B, Lemma 1, and Inequality C, we have

$$
\begin{aligned}
\prod_{\alpha}\left|f^{\alpha}(t)\right|^{q_{\alpha}} & \leq \frac{1}{q} \sum_{\alpha} q_{\alpha}\left|f^{\alpha}(t)\right|^{q} \leq \frac{1}{q} \sum_{\alpha} q_{\alpha}\left[\frac{1}{2 n} \sum_{i} \int_{a_{i}}^{b_{i}}\left|f_{i}^{\alpha}\right| d u_{i}\right]^{q} \\
& \leq \frac{1}{q} \sum_{\alpha} q_{\alpha}\left(\frac{1}{2 n}\right)^{q}\left[c(q, n) \sum_{i}\left(\int_{a_{i}}^{b_{i}}\left|f_{i}^{\alpha}\right| d u_{i}\right)^{q}\right] .
\end{aligned}
$$

Since $q>1$, we have $c(q, n)=n^{q-1}$. By Hölder's inequality,

$$
\begin{aligned}
\prod_{\alpha}\left|f^{\alpha}(t)\right|^{q_{\alpha}} & \leq \frac{1}{n q}\left(\frac{1}{2}\right)^{q} \sum_{\alpha, i} q_{\alpha}\left(\int_{a_{i}}^{b_{i}}\left|f_{i}^{\alpha}\right| d u_{i}\right)^{q} \\
& \leq \frac{1}{n q}\left(\frac{1}{2}\right)^{q} \sum_{\alpha, i} q_{\alpha}\left[\left(b_{i}-a_{i}\right)^{(q-1) / q}\left(\int_{a_{i}}^{b_{i}}\left|f_{i}^{\alpha}\right|^{q} d u_{i}\right)^{1 / q}\right]^{q} \\
& \leq \frac{1}{n q}\left(\frac{1}{2}\right)^{q} M^{q-1} \sum_{\alpha, i} q_{\alpha} \int_{a_{i}}^{b_{i}}\left|f_{i}^{\alpha}\right|^{q} d u_{i}
\end{aligned}
$$


Therefore,

$$
\begin{aligned}
\int \prod_{\alpha}\left|f^{\alpha}(t)\right|^{q_{\alpha}} d t & \leq \frac{1}{n q}\left(\frac{1}{2}\right)^{q} M^{q-1} \sum_{\alpha, i} q_{\alpha} \int_{\Omega} \int_{a_{i}}^{b_{i}}\left|f_{i}^{\alpha}\right|^{q} d u_{i} d t \\
& =\frac{1}{n q}\left(\frac{1}{2}\right)^{q} M^{q-1} \sum_{\alpha, i} q_{\alpha} \int_{a_{i}}^{b_{i}} \int_{\Omega}\left|f_{i}^{\alpha}\right|^{q} d t d u \\
& =\frac{1}{n q}\left(\frac{1}{2}\right)^{q} M^{q-1} \sum_{\alpha, i} q_{\alpha}\left(b_{i}-a_{i}\right) \int_{\Omega}\left|f_{i}^{\alpha}\right|^{q} d t \\
& \leq \frac{1}{n q}\left(\frac{M}{2}\right)^{q} \sum_{\alpha} q_{\alpha} \int_{\Omega} \sum_{i}\left|f_{i}^{\alpha}\right|^{q} d t \\
& =\frac{1}{n q}\left(\frac{M}{2}\right)^{q} \sum_{\alpha} q_{\alpha} \int_{\Omega}\left[\left(\sum_{i}\left|f_{i}^{\alpha}\right|^{q}\right)^{2 / q}\right]^{q / 2} d t \\
& \leq \frac{1}{n q}\left(\frac{M}{2}\right)^{q} \sum_{\alpha} q_{\alpha} \int_{\Omega}\left[c\left(\frac{2}{q}, n\right) \sum_{i}\left(\left|f_{i}^{\alpha}\right|^{2}\right)^{2 / q}\right]^{q / 2} d t
\end{aligned}
$$

by Inequality C. Since $q \geq 2$, we have $c\left(\frac{2}{q}, n\right)=1$ and so

$$
\begin{aligned}
\int_{\Omega} \prod_{\alpha}\left|f^{\alpha}(t)\right| d t & \leq \frac{1}{n q}\left(\frac{M}{2}\right)^{q} \sum_{\alpha} q_{\alpha} \int_{\Omega}\left(\sum_{i}\left|f_{i}^{\alpha}\right|^{2}\right)^{q / 2} d t \\
& =\frac{1}{n}\left(\frac{M}{2}\right)^{q} \sum_{\alpha} \frac{q_{\alpha}}{q} \int_{\Omega}\left|\nabla f^{\alpha}\right|^{q} d t . \text { Q.E.D. }
\end{aligned}
$$

Corollary 1. If $f^{\alpha} \in C_{0}^{1}(\Omega)$, then

$$
\int_{\Omega} \prod_{\alpha}\left|f^{\alpha}\right| \leq \frac{1}{n m} \sum_{\alpha}\left(\frac{M}{2}\right)^{m} \int_{\Omega}\left|\nabla f^{\alpha}\right|^{m} .
$$

Proof. This follows from Theorem 1 by setting $p_{\alpha}=m$ for all $\alpha$ or from Theorem 2 by setting $q_{\alpha}=1$ for all $\alpha$. Q.E.D.

Corollary 2. If $f \in C_{0}^{1}(\Omega)$, then

$$
\int_{\Omega}|f|^{m} \leq \frac{1}{n}\left(\frac{M}{2}\right)^{m} \int_{\Omega}|\nabla f|^{m}
$$

Proof. This follows from Corollary 1 by setting $f^{\alpha}=f$ for all $\alpha$. Q.E.D.

Remark. Notice that when $m=n=2$ and $\Omega$ is a square, Corollary 2 is sharper than the result in [7] in the sense that our constant here is $M^{2} / 8$, comparing to $7 M^{2} / 12$ there.

Corollary 3. If $f^{\alpha} \in C_{0}^{1}(\Omega)$ and $p_{\alpha} \geq 2$ are real numbers satisfying $\sum_{\alpha} 1 / p_{\alpha}=$ 1 , then

$$
\int_{\Omega} \sum_{\beta} \prod_{\alpha \neq \beta}\left|f^{\alpha} \nabla f^{\beta}\right| \leq\left(\frac{M}{2}\right)^{m-1}\left[\sum_{\alpha}\left(\frac{1}{n}\right)^{1-1 / p_{\alpha}}\right]\left[\sum_{\alpha} \frac{1}{p_{\alpha}} \int_{\Omega}\left|\nabla f^{\alpha}\right|^{p_{\alpha}}\right] .
$$


Proof. By Inequality D and Corollary 2, we have

$$
\begin{aligned}
\int_{\Omega} \sum_{\beta} \prod_{\alpha \neq \beta}\left|f^{\alpha} \nabla f^{\beta}\right| \\
\leq \sum_{\beta}\left[\prod_{\alpha \neq \beta}\left(\int_{\Omega}\left|f^{\alpha}\right|^{p_{\alpha}}\right)^{1 / p_{\alpha}}\left(\int_{\Omega}\left|\nabla f^{\beta}\right|^{p_{\beta}}\right)^{1 / p_{\beta}}\right] \\
\leq \sum_{\beta}\left[\prod_{\alpha \neq \beta}\left(\frac{1}{n}\left(\frac{M}{2}\right)^{p_{\alpha}} \int_{\Omega}|\nabla f|^{p_{\alpha}}\right)^{1 / p_{\alpha}}\left(\int_{\Omega}\left|\nabla f^{\beta}\right|^{p_{\beta}}\right)^{1 / p_{\beta}}\right] \\
\quad=\left[\sum_{\beta}\left(\frac{1}{n}\right)^{1-1 / p_{\beta}}\left(\frac{M}{2}\right)^{m-1}\right] \prod_{\alpha}\left(\int_{\Omega}\left|\nabla f^{\alpha}\right|^{p_{\alpha}}\right)^{1 / p_{\alpha}} .
\end{aligned}
$$

Therefore, by Inequality $B$, we arrive at

$$
\begin{aligned}
& \int_{\Omega} \sum_{\beta} \prod_{\alpha \neq \beta}\left|f^{\alpha} \nabla f^{\beta}\right| \\
& \leq\left[\sum_{\beta}\left(\frac{1}{n}\right)^{1-1 / p_{\beta}}\left(\frac{M}{2}\right)^{m-1}\right] \cdot \frac{1}{\sum\left(1 / p_{\beta}\right)} \sum_{\alpha} \frac{1}{p_{\alpha}}\left(\int_{\Omega}\left|\nabla f^{\alpha}\right|^{p_{\alpha}}\right)^{\sum\left(1 / p_{\beta}\right)} \\
& \quad=\left(\frac{M}{2}\right)^{m-1}\left[\sum_{\alpha}\left(\frac{1}{n}\right)^{1-1 / p_{\alpha}}\right]\left[\sum_{\alpha} \frac{1}{p_{\alpha}} \int_{\Omega}\left|\nabla f^{\alpha}\right|^{p_{\alpha}}\right] \text {. Q.E.D. }
\end{aligned}
$$

Corollary 4. If $f^{\alpha} \in C_{0}^{1}(\Omega)$, then

$$
\int_{\Omega} \sum_{\beta} \prod_{\alpha \neq \beta}\left|f^{\alpha} \nabla f^{\beta}\right| \leq\left(\frac{M}{2}\right)^{m-1}\left(\frac{1}{n}\right)^{1-1 / m} \sum_{\alpha} \int_{\Omega}\left|\nabla f^{\alpha}\right|^{m} .
$$

Proof. This follows immediately from Corollary 3 by setting $p_{\alpha}=m$ for all $\alpha$. Q.E.D.

Remark. Further interesting integral inequalities of the Poincaré-type could easily be generated from the results above. For instance, by taking $m=2$ in Corollary 4 , we get

$$
\int_{\Omega}|f \nabla g+g \nabla f| \leq \frac{M}{2 \sqrt{n}}\left(\int_{\Omega}|\nabla f|^{2}+\int_{\Omega}|\nabla g|^{2}\right),
$$

and by putting $f=g$ in the last expression, we have

$$
\int_{\Omega}|f \nabla f| \leq \frac{M}{2 \sqrt{n}} \int_{\Omega}|\nabla f|^{2} .
$$

These integral inequalities are in general of great interest and have been proven to be very useful in the study of quantitative as well as qualitative properties of solutions of many differential and integral equations.

\section{REFERENCES}

1. E. F. Beckenbach and R. Bellman, Inequalities, Springer-Verlag, New York, 1965.

2. W. S. Cheung, Some new Opial-type inequalities, Mathematika 37 (1990), 136-142. 
3. G. H. Hardy, J. E. Littlewood, and G. Pólya, Inequalities, Cambridge Univ. Press, Cambridge, 1952.

4. C. O. Horgan, Integral bounds for solutions of nonlinear reaction-diffusion equations, J. Appl. Math. Phys. (ZAMP) 28 (1977), 197-204.

5. C. O. Horgan and R. R. Nachlinger, On the domain of attraction for steady states in heat conduction, Internat. J. Engrg. Sci. 14 (1976), 143-148.

6. C. O. Horgan and L. T. Wheeler, Spatial decay estimates for the Navier-Stokes equations with applications to the problem of entry flow, SIAM J. Appl. Math. 35 (1978), 97-116.

7. D. S. Mitrinović, Analytic inequalities, Springer-Verlag, Berlin, 1970.

8. L. Nirenberg, On elliptic partial differential equations, Ann. Scuola Norm. Sup. Pisa Cl. Sci. (4) 13 (1959), 116-162.

9. B. G. Pachpatte, On Poincaré-type integral inequalities, J. Math. Anal. Appl. 114 (1986), 111-115.

10. $\ldots$, On some new integral inequalities in several independent variables, Chinese J. Math. 14 (1986), 69-79.

Department of Mathematics, University of Hong Kong, Pokfulam Road, Hong Kong E-mail address: wscheung@hkucc.hku.hk 